\title{
Adaptive Modulation Amplitude in 2D Spectral-Spatial EPR Imaging
}

\author{
T. Czechowski ${ }^{a, b}$, A. Samolej ${ }^{b}$, M. Baranowski $^{a, c, d}$, A. Boś-Liedke ${ }^{a, e}$, W. Chlewicki $^{d, e}$, \\ J. JURGA ${ }^{b, e}$ AND K. TADYSZAK ${ }^{a, f, *}$ \\ ${ }^{a}$ NanoBioMedical Centre, Adam Mickiewicz University, Poznań, Poland \\ ${ }^{b}$ Laboratory of EPR Tomography, Poznan University of Technology, Poznań, Poland \\ ${ }^{c}$ Department of Physics, Faculty of Physics, Adam Mickiewicz University, Poznań, Poland \\ ${ }^{d}$ Faculty of Electrical Engineering, West Pomeranian University of Technology, Szczecin, Poland \\ ${ }^{e}$ noviLET, Poznań, Poland \\ ${ }^{e}$ Department of Medical Physics, Faculty of Physics, Adam Mickiewicz University, Poznań, Poland \\ ${ }^{f}$ Institute of Molecular Physics Polish Academy of Sciences, Poznań, Poland
}

A study concerning the image quality in Electron Paramagnetic Resonance Imaging (EPRI) in 2D spectralspatial (2D SSI) experiments is presented. The aim of the measurements is to improve the signal to noise ratio (SNR) of the projections by applying a more consciously selected modulation amplitude parameter. Data is gathered by applying three constant and one adaptive modulation amplitude. The three fixed modulation amplitudes values are leading to undermodulated $(0.01 \mathrm{G})$, partially overmodulated $(0.15 \mathrm{G})$ and fully overmodulated $(0.65 \mathrm{G})$ projections. The study demonstrates the advantages of the adaptive method, which involves selecting different and dependent on cosine function modulation amplitudes for each projection. The study is performed on a phantom containing four tubes of LiPc and TCNQ, characterized by a different peak to peak linewidth and spin concentration.

DOI: 10.12693/APhysPolA.133.710

PACS/topics: 76.30.-v; 42.30.Wb; 75.20.-g; 42.30.Va

\section{Introduction}

The Electron Paramagnetic Resonance Imaging (EPRI) is a method of spatially and spectrally resolving signals resulting from unpaired electrons in the sample, by applying magnetic field gradients. Depending on the method and reconstruction algorithm, it is possible to differentiate two types of imaging: the spatial-spatial and the spectral-spatial imaging. In the first case, only the information about the spatial radical concentration can be gained, in second type also the spectral features of the signal (e.g. linewidth, isotropic Fermi coupling constants) are available for each voxel. The image in spatial imaging is reconstructed from a set of projections recorded at the same magnetic field gradient strength, but rotated stepwise in between scans for each projection, while EPR signal is detected [1]. In case of spectralspatial imaging the gradient strength is also changing. The importance of spectral-spatial experiments lies in the possibility to measure oxygen partial pressure $\mathrm{O}_{2}$, which is one of the most important measurements in biology, medicine [2-5], as well other parameters like $\mathrm{pH}[6]$, redox status [7], thiols [46], nitric oxide - NO [8]. To obtain high spatial resolution in EPRI experiments large magnetic field gradient needs to be applied, which unfortunately causes signal broadening with simultaneous

*corresponding author; e-mail: t.czechowski@novilet.eu amplitude decrease. Multiple attempts were made to increase the signal to noise ratio (SNR), which resulted in increasing the measurement speed $[9,10]$, adjusting the detection method $[1,11,12]$ and spectral-spatial imaging technique [13]. To overcome low SNR the amplitude of modulation higher than the $1 / 3$ of the linewidth (referred to as an overmodulation) has been proposed. Unfortunately using too high modulation amplitudes distorts the line shape and additional signal restoring procedures needs to be applied [14-16]. At the same time, there are no articles which analyze the effects of using partially and fully overmodulated projections to image quality. This article compares image qualities of four 2D spectral-spatial EPR images of a LiPc and TCNQ phantom which is recorded with different constant modulation amplitudes and one dependent on gradient strength (adaptive method).

\section{Materials and methods}

The measurements were carried out using E540 L-Band Bruker Spectrometer equipped with E 540R23 L-Band EPR-Resonator. Projections were recorded in the angle range $-88^{\circ}(-20 \mathrm{G} / \mathrm{cm}$, left side Fig. $1 \mathrm{~b})$ to $88^{\circ}(20 \mathrm{G} / \mathrm{cm}$, right side Fig. 1b) with step $4^{\circ}$. The gradient field direction was constant during the entire experiment, giving in total 45 projections. Number of projection was set by the equation, where $i$ is an iterator defined as $i=(0 \ldots 44)$. Each projection was recorded with its own sweep width, depending on the viewing angle which depends on the 
spectral image size $(\Delta B=1 \mathrm{G})$, and the spatial window $(L=15 \mathrm{~mm})$, by the equations $\tan (\alpha)=\frac{L G}{\Delta B}$ and sweep $=\sqrt{2} \Delta B / \cos (\alpha)$. The rest of parameters were: microwave power $0.11 \mathrm{~mW}$, initial field sweep $40.52 \mathrm{G}$, projection points 1024 , frequency $1.096 \mathrm{GHz}$. Measurements were performed for three different constant modulation amplitudes $0.01,0.15,0.65 \mathrm{G}$ and the adaptive one, in which the modulation amplitude is defined as $A / \cos (\alpha)$, where $A$ is equal to 0.01 , which is less than $1 / 3$ of the smallest linewidth, and $\alpha$ is the projection angle. In all cases the time of measurement was the same. During the tests stable radicals like lithium phthalocyanine $(\mathrm{LiPc})$ and TCNQ anion salt were used. Phantom was made out of four tubes set in a row (Fig. 1a). Crystallites of $\mathrm{LiPc}$ were measured in $3 \mathrm{~mm}$ (outer diameter) tubes with wall thickness $0.75 \mathrm{~mm}$, and TCNQ anion radicals in $1.7 \mathrm{~mm}$ (outer diameter) tubes with wall thickness $0.35 \mathrm{~mm}$. The distances between the main axes of the tubes were $2.35,3$, and $2.35 \mathrm{~mm}$. The orginal peak to peak linewidths of LiPs are $68 \mathrm{mG}\left(8.4 \times 10^{16}\right.$ spins $)$ and $97 \mathrm{mG}\left(1.2 \times 10^{17}\right.$ spins $)$, and TCNQ are $370 \mathrm{mG}$ $\left(1.8 \times 10^{17}\right.$ and $4.5 \times 10^{17}$ spins $)$. The SNR is defined as the peak to peak amplitude of the signal divided by the standard deviation measured at the beginning of the spectra.

\section{Results and discussion}

In the spectral-spatial imaging technique the most informative projections are the ones measured at the highest magnetic field gradients and simultaneously they are the ones having lowest amplitudes and strongest signal broadening due to the magnetic field gradient applied.

To investigate the influence of modulation amplitude on the reconstructed images four cases of different modulation amplitudes were considered and analyzed. At first, the modulation amplitude was less than $1 / 3$ of the smallest linewidth $(6.8$ times less $-0.01 \mathrm{G})$, second was approximately 2.2 times higher than smallest linewidth $(0.15 \mathrm{G})$, third was almost 9.6 times higher than the smallest linewidth $(0.65 \mathrm{G})$, and finally, the adaptive amplitude for each projection, described by the function $A_{\text {mod }}=0.01 / \cos (\alpha)$, giving the values always smaller than $1 / 3$ of the linewidth of the gradient broadened spectrum (projection linewidth). This linewidth is defined as the width between first maximum and the last minimum of the gradient broadened, first derivative spectrum. This method is based on the increase of modulation amplitude with the increase of gradient strength. It is our assumption that when the projection linewidth increases due to the gradient strength the modulation amplitude can be increased to some extent as well. At the same time, this procedure can lead to image distortions, unless the maximum modulation amplitude is set lower than $1 / 3$ of the gradient broadened linewidth (chosen arbitrary), than the distortions do not appear. This method provides projections with higher SNR and also a correct image reconstruction without the use of spectral fitting.

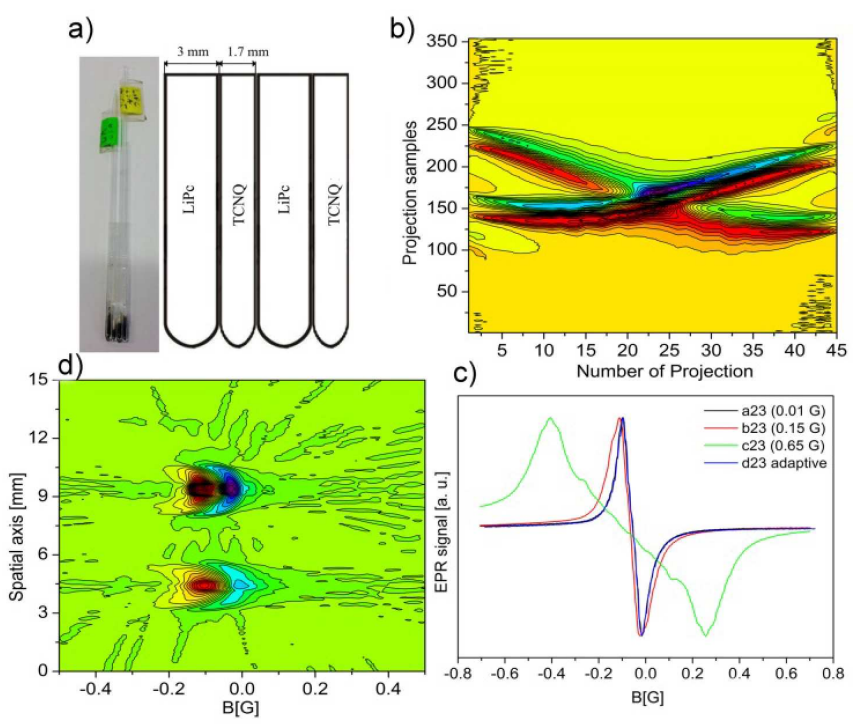

Fig. 1. a) phantom; b) sinogram - adaptive method; c) sinograms cross sections; d) reconstructed 2D image.

Figure 1a shows the phantom made of four tubes, which was placed in the resonator and recorded with fixed gradient direction parallel to the external magnetic field for 45 different gradient strengths. The top view of all projections is showed in Fig. 1b. After image reconstruction by filtered back projection (FBP) method the final image is obtained (Fig. 1d). The same procedure was applied to all recordings giving in total four sinograms and four images out of them. The normalized cross sections taken at 23 projection is showed at Fig. 1c. Only two signals for $0.01 \mathrm{G}$ and from adaptive method are not initially broadened, giving the possibility of correct image reconstruction without additional steps. Although the SNR in the case $0.65 \mathrm{G}$ is the highest, strong line deformation makes it mandatory to use line reconstruction as a next step extending the time and effort necessary for the image reconstruction.

To compare the reconstruction quality in each case the peak-to-peak linewidth (e.g. Fig. 1d) was plotted against spatial position of the sample and summarized in Table I. The error estimation of linewidths is handled by sum of relative errors and $\chi$-square coefficient defined as: $\sum_{\text {r.e. }}=\sum_{i=1}^{4} \frac{\left|\Delta B_{i}^{\text {exp }}-\Delta B_{i}^{\text {or } i}\right|}{\Delta B_{i}^{\text {ori }}}$, where $\Delta B_{i}^{\text {exp }}$ is experimental linewidth, and $\Delta B_{i}^{\text {ori }}$ is the original linewidth. The best result was obtained with the adaptive method where $\chi$-square is 23 , while the worst result - 16.1 and 8918 was obtained in the overmodulated case $(0.65 \mathrm{G})$. Three out of four linewidths with smallest errors belong to the adaptive method (underlined linewidths in Table I).

The fitting to overmodulated data (Robinson model) was second in order with the result 0.96 and 149 , and one linewidth measurement with smallest error. The fit to adaptive method was before last (1.8 and 330). The Robinson model showed its full potential in the overmodulated case where signal was the strongest. Enormous 


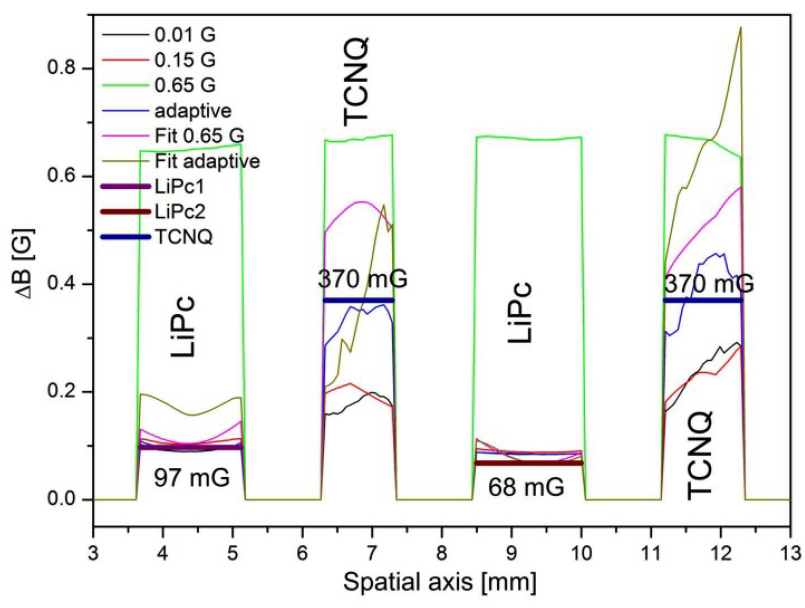

Fig. 2. Linewidth vs. spatial axis position for four cases. Thick lines show the expected values.

TABLE I

Linewidths and errors adapted from Fig. 2. Underlined numbers are the best experimental result for specific sample.

\begin{tabular}{c|c|c|c|c|c|c|c}
\hline \hline \multirow{2}{*}{ Sample } & \multirow{2}{*}{$B^{\text {ori }}$} & \multicolumn{5}{|c}{ Experimental linewidth [mG] } \\
\cline { 3 - 8 } & {$[\mathrm{mG}]$} & \multicolumn{4}{|c}{ Case } & \multicolumn{2}{c}{ Fit to } \\
\cline { 3 - 8 } & & $0.01 \mathrm{G}$ & $0.15 \mathrm{G}$ & $0.65 \mathrm{G}$ & adapt. & $0.65 \mathrm{G}$ & adapt. \\
\hline LiPc & 97 & 89 & 102 & 650 & $\underline{97}$ & 104 & 157 \\
$\mathrm{TCNQ}$ & 370 & 196 & 191 & 672 & $\underline{346}$ & 550 & 510 \\
$\mathrm{LiPc}$ & 68 & 84 & 88 & 670 & 84 & $\underline{68}$ & 67 \\
$\mathrm{TCNQ}$ & 370 & 260 & 233 & 635 & $\underline{450}$ & 520 & 668 \\
$\sum_{\substack{\text { column } \\
\sum}}$ & 905 & 629 & 614 & 2627 & 977 & 1242 & 1402 \\
$\frac{\text { column }}{905}$ & 1 & 0.70 & 0.68 & 2.90 & 1.08 & 1.37 & 1.55 \\
$\sum_{r . e .}$ & - & 1.09 & 1.2 & 16.1 & 0.52 & 0.96 & 1.8 \\
$\chi^{2}$ & 0 & 119 & 144 & 8918 & 23 & 149 & 330
\end{tabular}

improvement was achieved in the case of Fit $0.65 \mathrm{G}$, compared to the original value $(0.65 \mathrm{G})$ - a decrement in total linewidth of 2.1 times $(2627 / 1242)$ was reported.

\section{Conclusions}

Adaptive method which modifies the modulation amplitude in each projection by making it inversely dependent on a cosine function of the projection angle showed an improvement of the SNR for higher projection angles in comparison to the undermodulated case (0.01 $\mathrm{G})$. Adaptive method showed above five times smaller $\chi$-square value after reconstruction than it was observed in the undermodulated case. The strongest advantage of this method is that it does not require a spectral fitting step after image reconstruction, unlike the overmodulation case.

\section{Acknowledgments}

Financial support from the National Science Centre (UMO-2014/15/B/ST4/04946), National Cohesion Strategy from Innovative Economy (POIG.01.03.01-30150/09) and Smart Growth (POIR.01.01.01-00-0025/15) is gratefully acknowledged. Lithium phthalocyanine is a generous gift from Dr. Harold Swartz, Department of Radiology, Dartmouth College. The authors wish to thank Dr. Emerson Coy from NanoBioMedical Center and Zuzanna Kabacińska from Department of Medical Physics, Faculty of Physics, Adam Mickiewicz University for their help and fruitful discussions during the development of this work.

\section{References}

[1] T. Czechowski, et al., J. Magn Reson. 248, 126 (2014).

[2] R. Mrowczynski, et al., J Phys Chem B. 119, 10341 (2015).

[3] M. Elas, et al., Magn Reson Med. 49, 682 (2003).

[4] S. Liu, et al., NMR Biomed. 17, 327 (2004).

[5] J. Weaver, et al., Toxicol Appl Pharmacol. 275, 73 (2014).

[6] S. Kempe, et al., Eur J Pharm Biopharm. 74, 55 (2010).

[7] P. Kuppusamy, M. Krishna, Curr. Top. Biophys. 26 29 (2002).

[8] P. Kuppusamy, et al., J Cereb Blood Flow Metab. 15, 899 (1995).

[9] T. Czechowski, et al., Applied Magnetic Resonance. 29, 335 (2005).

[10] K. Tadyszak, et al., Magn Reson Chem. 54, 136 (2016).

[11] T. Czechowski, et al., Conc. Magn. Reson. B: Magn. Reson. Eng. 43B, 22 (2013).

[12] T. Czechowski, et al., J Magn Reson. 243, 1 (2014).

[13] G.R. Eaton, et al., Int. J. Rad. Appl. Instr. A. Appl. Rad. Isot. 40, 1227 (1989).

[14] B.B. Williams, et al., Antioxid. Redox Signal. 9, 1691 (2007).

[15] Y. Deng, et al., J. Magn. Res. 181, 254 (2006).

[16] B.H. Robinson, A.W.R.C. Mailer, J. Magn. Res. 138, 199 (1999). 